\title{
Transport Length Scales in Disordered Graphene-based Materials: Strong Localization Regimes and Dimensionality Effects
}

\author{
Aurélien Lherbier ${ }^{1,3}$, Blanca Biel ${ }^{2}$, Yann-Michel Niquet ${ }^{3}$ and Stephan Roche ${ }^{4}$ \\ 1 Laboratoire des Technologies de la Microlectronique (LTM), \\ UMR 5129 CNRS, CEA 17 Rue des Martyrs 38054 Grenoble France \\ 2 CEA, LETI-MINATEC, 17 rue des Martyrs, 38054 Grenoble, Cedex 9 France \\ 3 CEA, Institut des Nanosciences et Cryogénie, SP2M/L_Sim, \\ 17 rue des Martyrs, 38054 Grenoble Cedex 9, France \\ 4 CEA, Institut des Nanosciences et Cryogénie, SPSMS/GT, \\ 17 rue des Martyrs, 38054 Grenoble Cedex 9, France
}

(Dated: October 20, 2018)

\begin{abstract}
We report on a numerical study of quantum transport in disordered two dimensional graphene and graphene nanoribbons. By using the Kubo and the Landauer approaches, transport length scales in the diffusive (mean free path, charge mobilities) and localized regimes (localization lengths) are computed, assuming a short range disorder (Anderson-type). In agreement with localization scaling theory, the electronic systems are found to undergo a conventional Anderson localization in the zero temperature limit. Localization lengths in weakly disordered ribbons are found to differ by two orders of magnitude depending on their edge symmetry, but always remain several orders of magnitude smaller than those computed for 2D graphene for the same disorder strength. This pinpoints the role of transport dimensionality and edge effects.
\end{abstract}

PACS numbers: 73.63.-b,72.15.Rn,81.05.Uw 
Recently, single graphene sheet could be isolated either from chemical exfoliation of bulk graphite [1], or by epitaxial growth on metal substrates through thermal decomposition of $\mathrm{SiC}[2]$. These technological achievements have opened unprecedented opportunities to explore quantum transport in low dimensional carbon-based disordered systems [3, 4].

Because of the unique electronic properties of the 2D graphene (massless Dirac fermions with linear dispersion and electron-hole symmetry), disorder effects and transport properties turn out to be unconventional. Theoretically, it has been shown that for long range impurity potentials, intervalley $K \rightarrow K^{\prime}$ scattering between the two Dirac nodes could be strongly reduced, resulting in anomalously low backscattering rates [5], extremely large elastic mean free paths and vanishingly small localization effects [6]. In contrast, for short range impurity potentials (where all types of scattering between $K$ and $K^{\prime}$ are allowed), stronger quantum interferences could develop, leading to weak localization, or strong Anderson localization in the zero-temperature limit [7]. To date magnetotransport experiments either performed on exfoliated or epitaxial graphene have reported both weak antilocalization and weak localization effects [8], confirming the sensitivity of 2D transport in graphene to the external random potential, whose precise origin remains unknown.

Beyond 2D graphene physics, the transport properties of quasi-1D graphene nanoribbons (GNRs) with width down to a few tens of nanometers have been characterized [9]. In contrast to 2D graphene, the electronic properties of GNRs are strongly dependent on confinement effects and edge symmetries [10]. These new structures share similarities with carbon nanotubes, often viewed as rolled single graphene ribbons, and that have provided unique materials for investigating 1D transport phenomena such as Luttinger liquid and Kondo physics or Anderson localization [11].

The issue of localization in graphene-based materials is currently highly debated from a theoretical standpoint. For instance, the measurement of a minimal conductivity of $\sim$ $2-5 e^{2} / h$ in samples for which charge mobilities change, however, by almost one order of magnitude remains to be fully understood [1, 2, 3]. Indeed, a conventional treatment of disorder effects within the self consistent Born approximation (SCBA) yields $\sigma_{x x}^{\min } \sim$ $4 e^{2} /(h \pi)$ for the two Dirac nodes [5] ( $h$ the Planck constant), hence typically smaller by a $1 / \pi$ factor with respect to the experimental data. Depending on the disorder model, the use of the Kubo approach suggest several scenarii to understand such discrepancies [12]. Besides, the role played by quantum interferences and the transition to a localization regime 
in graphene and GNRs remain poorly explored but fiercely debated [7].

In this Letter, by using both the Kubo and Landauer approaches, the transport length scales in 2D graphene are investigated and compared with those of the quasi-1D GNRs. The disorder (Anderson-type) is introduced via random fluctuations of the onsite energies of the $\pi$-orbitals, which mimics a short range scattering potential that has been widely studied in the past as a generic disorder model in the framework of localization theory [13, 14]. For 2D graphene, a real space order $N$ Kubo method [16] is used to compute the energy-dependent elastic mean free path $\left(\ell_{e}\right)$, charge mobilities $(\mu)$ and semiclassical conductivities $\left(\sigma_{\mathrm{sc}}\right)$ in the diffusive regime, before quantum interferences come into play. Beyond the diffusive regime, the energy-dependent localization length $(\xi)$ is extracted from the analysis of the transition from weak to strong localization, following the scaling theory phenomenology [14]. Quantum transport in GNRs with different chiralities (ziz-zag and armchair type) and same disorder potential is also investigated within a Landauer approach [15]. For GNRs widths in the range $\sim 20-80 \mathrm{~nm}$ (within the experimental scope [9]), it is found that edge effects strongly enhance the impact of disorder, which results in localization lengths several orders of magnitude smaller than those obtained in 2D graphene for the same disorder strength.

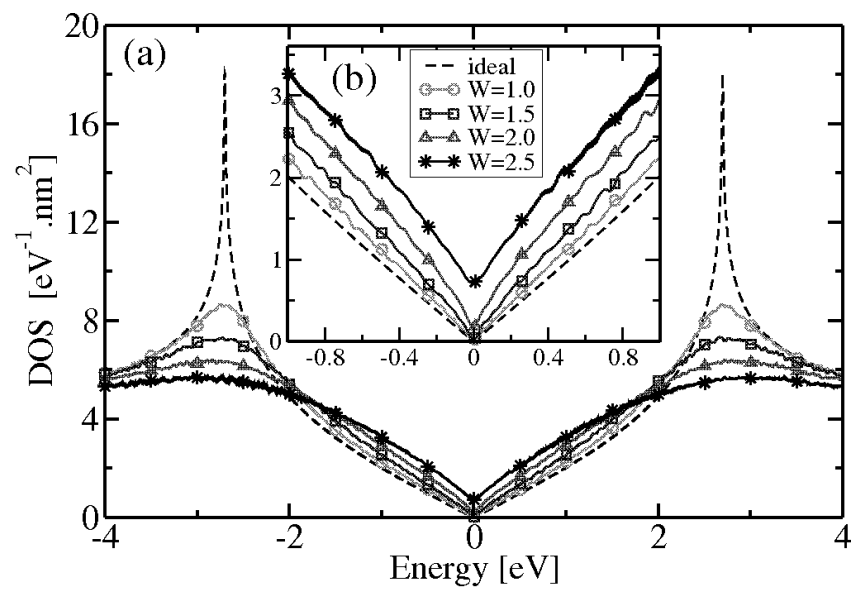

FIG. 1: (color online) (a): DoS of an ideal (dashed lines) and for disordered graphene sheets for several values of $W=1,1.5,2,2.5$ (b): Zoom in the energy area around the CNP.

The low energy electronic properties of $2 \mathrm{D}$ graphene are accurately described by the $\pi$ orbital tight-binding hamiltonian, which is a first nearest neighbor two centers orthogonal $p_{z}$ model, with onsite energies $\varepsilon_{c}=0 \mathrm{eV}$ for all orbitals and hopping term $\gamma_{0}=2.7 \mathrm{eV}$. To mimic short range disorder, a white noise uncorrelated Anderson type disorder is introduced as a 
random fluctuation of the onsite energies of the hamiltonian $\left(\varepsilon=\varepsilon_{c}+\delta \varepsilon\right)$. The scattering potential can thus be characterized by a single parameter $W$ which defines the range of energy variations $\left(\delta \varepsilon \in\left[-W \gamma_{0} / 2,+W \gamma_{0} / 2\right]\right)$, and thus allows to tune the disorder strength. In what follows $W=[0.5,2.5]$ enables the exploration of all transport regimes taking place in disordered 2D graphene and GNRs.

In Fig. 1, the Density of States (DoS), computed with a Lanczos-type method [16], is reported as a function of disorder strength. The disorder-free DoS (dashed line) shows the typical behavior with a linear increase at low energy and the presence of two sharp Van Hove singularities at $E= \pm \gamma_{0}$. As $W$ is increased, two opposite behaviors are observed. At high energies, Van Hove singularities are smoothen whereas close to the charge neutrality point (CNP), disorder enhances the DoS in agreement with prior analytical results [5] (see Fig. 1b for a close-up).

To investigate quantum transport in the $2 \mathrm{D}$ disordered graphene, an efficient real space and order $N$ Kubo method is employed [16]. In this formalism, the mean free path $\ell_{e}(E)$, the semiclassical conductivity $\sigma_{s c}(E)$ and the charge carrier mobility $\mu(E)$ are deduced from the energy and time dependence of the diffusion coefficient $D(E, t)=\left\langle\Delta R^{2}\right\rangle(E, t) / t$ (where $\left\langle\Delta R^{2}\right\rangle(E, t)$ is the quadratic spread of random phase wavepackets propagated in the graphene sheet).

In Fig. 2 2 , the time dependence of $D(E, t)$ at the CNP and at $E=0.1 \mathrm{eV}$ are reported for two values of $W$ [17]. Different transport regimes follow each other as a function of the propagation time (or length). As expected, $D(E, t)$ first scales linearly with $t$ at short times owing to the absence of elastic scattering. This linear scaling is followed by a saturation of $D$ at a maximum value $D_{\max }(E)$, that pinpoints the occurrence of a diffusive regime for which $D \sim v \ell_{e}$ (with $v$ a group velocity and $\ell_{e}$ the elastic mean free path [16]). As evidenced in Fig. 2k $k$, the saturation time decreases with increasing disorder strength $(W)$ or increasing charge energy $(\mathrm{E})$. At longer times, $D(E, t)$ decreases owing to quantum interferences effects and localization phenomena [16]. The full energy-dependence of $\ell_{e}$ is given in Fig. 2b for increasing $W$.

The strong enhancement of $\ell_{e}$ around the CNP results from the cusp in the DoS, which implies a reduced number of scattering processes. However, $\ell_{e}(E)$ drops from $180 \mathrm{~nm}$ at $W=1.0$ to $10 \mathrm{~nm}$ at $W=2.0$, as a consequence of the increase of the DoS close to CNP. In the weak disorder case ( $W \in[0.2-0.7]$ not shown here), when the DoS at the CNP is 


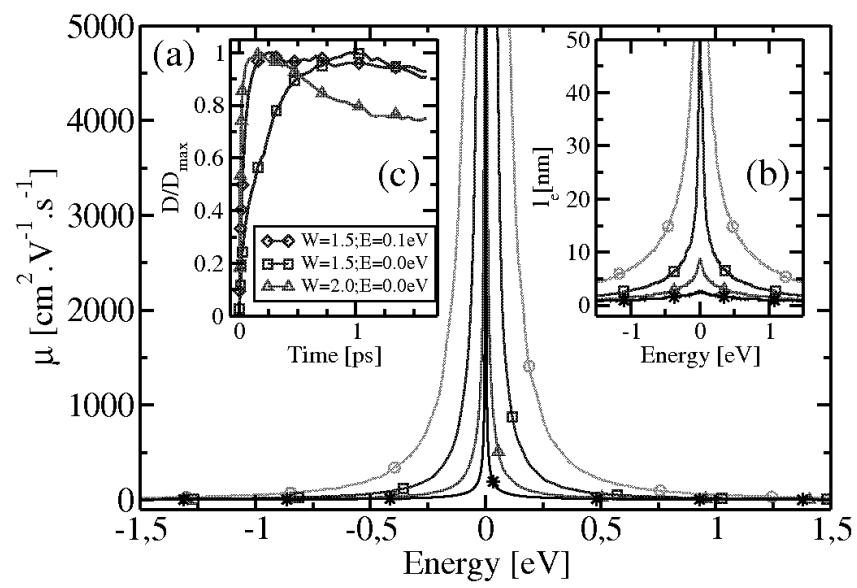

FIG. 2: (color online) (a): Energy-dependent charge mobility for several values of W. (b): $\ell_{e}$ for the same disorder strengths. The legend is the same as in Fig. 1. (c): Diffusion coefficient $D(E, t)$ as a function of time for various disorder strengths and Fermi energies. $D(E, t)$ has been normalized with respect to its maximum value $D_{\max }(E)$ to allow an easier comparison between the different curves.

almost unchanged with respect to disorder-free graphene case, the behavior of $\ell_{e}(E)$ as a function of $W$ is in good agreement with the Fermi golden rule (FGR) i.e. $\ell_{e}(E) \propto 1 / W^{2}$. For higher values of $W$ (1.0 to 2.0) slight deviations to the FGR are expected, since the weak disorder approximation is not strictly applicable anymore.

Fig. $2 \mathrm{a}$ shows the corresponding charge mobilities deduced from $\mu(E)=\sigma_{\mathrm{sc}}(E) / e n(E)$, where $\sigma_{\mathrm{sc}}=e^{2} \rho(E) v(E) \ell_{e}$ is the semiclassical conductivity deduced from the Einstein formula, $\rho(E)$ is the DoS, $n(E)$ is the charge density at energy $E$, and is $e$ the elementary charge. The energy-dependence of $\mu(E)$ and $\ell_{e}(E)$ are similar, and the sharp increase of $\mu(E)$ in the vicinity of CNP is in good qualitative agreement with experimental observations $[2,3,4]$.

Experiments show that the conductivity (down to a few Kelvin) is almost constant close to the CNP, $\sigma(E=0) \sim 3-5 e^{2} / h$, and weakly dependent on the value of the charge mobility [1, 2, 3]. On the theoretical side, within the SCBA the semiclassical part of the conductivity due to short range disorder is found to be $\sigma_{\mathrm{sc}}=4 e^{2} /(h \pi)[\underline{5}]$. By using the Kubo formalism, it was further found that $\sigma(E=0)$ strongly depends on the nature of the scattering potential (short or long range) [12]. Our numerical results are shown in Fig. 3b. $\sigma_{s c}$ clearly remains larger or equal to $\sigma_{\min }=2 G_{0} / \pi\left(G_{0}=2 e^{2} / h\right)$, a fact that would be 


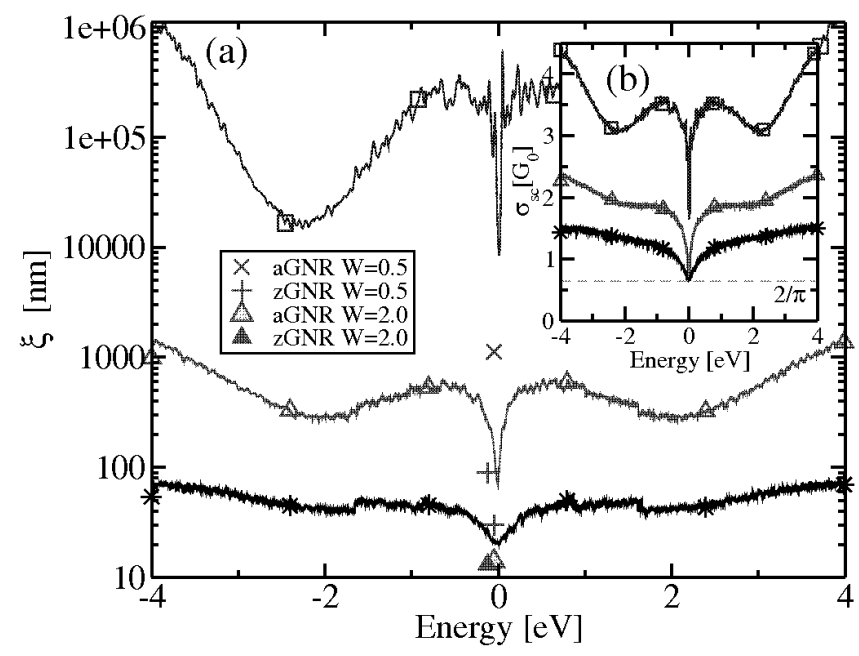

FIG. 3: (color online) (a): $\xi(E)$ for three disorder strengths (same legend as in Fig. 1). (b): Energy-dependent semiclassical conductivity for the same disorder strengths.

consistent with Mott argument [14], although localization effects are further observed at all energies (see hereafter). Besides, the shape of the energy-dependence of conductivity is in perfect agreement with prior analytical results derived for short range disorder within the SCBA [5].

However, the conductivity would not be sensitive to localization effects only in the presence of some decoherence mechanisms such as electron-electron scattering of electron-phonon coupling [14]. In contrast, as previously seen in the time-dependence of the diffusion coefficient (Fig. 2k), our zero-temperature calculations evidence the contribution of localization effects that develop beyond the diffusive regime. The $2 \mathrm{D}$ localization length $\xi$ can be evaluated as follows [14]: Whatever the disorder model, the quantum correction to the conductivity is expected to scale as $\Delta \sigma(L)=\left(G_{0} / \pi\right) \ln \left(L / \ell_{e}\right)$, where $L$ is the length scale associated with the propagation time. The localization length $\xi$ is given by $\Delta \sigma(L=\xi)=\sigma_{s c}$, i.e. $\xi=\ell_{e} \exp \left(\pi \sigma_{s c} / G_{0}\right)$. Our results are reported in Fig. 3a for several disorder strengths. The energy-dependence of $\xi$ is mainly dominated by that of $\sigma_{s c}$. As a result, although $\ell_{e}$ is strongly increasing as the Fermi level moves towards the CNP (undoped case), the behavior of $\xi$ shows an opposite trend, with a minimum value at CNP.

Recently, the possibility to fabricate quasi-1D graphene nanoribbons has opened new perspectives for future carbon-based nanoelectronics [2, 9]. It is thus important to evaluate the effects of disorder in this situation of lower dimensionality. Indeed, the bandstructures 


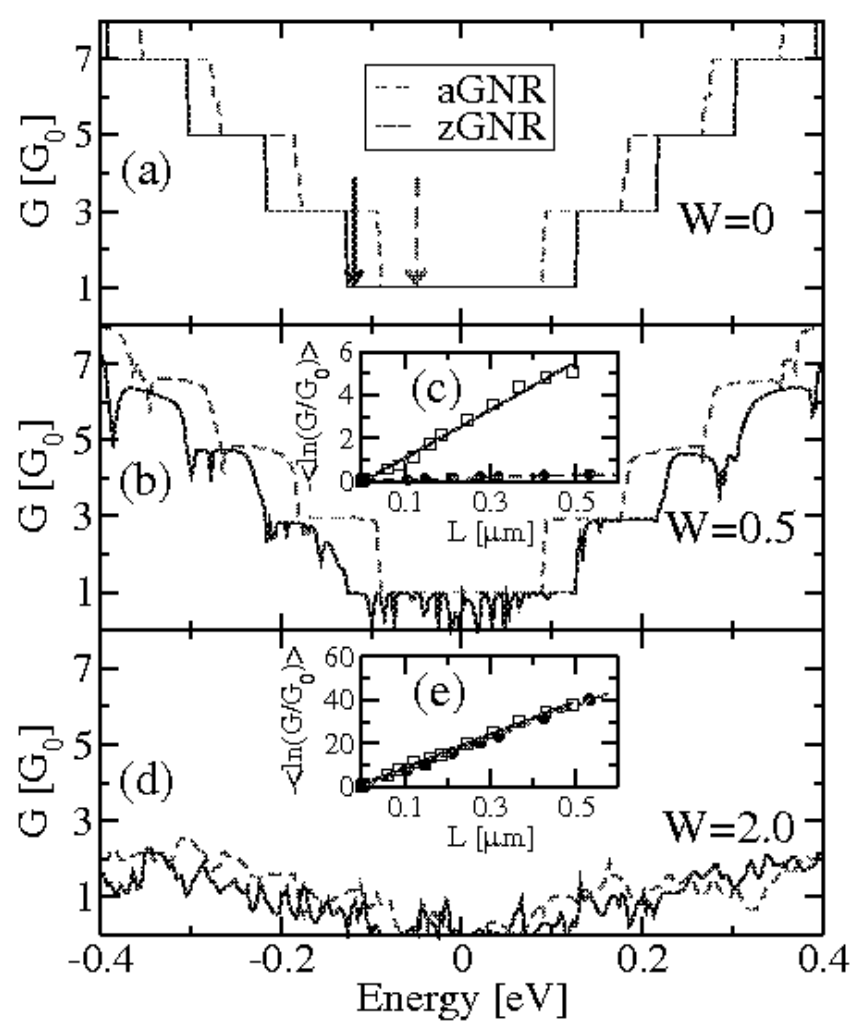

FIG. 4: (color online) (a): Conductance for ideal ziz-zag (solid blue line) and armchair (dashed red line) GNRs with width of $\sim 20 \mathrm{~nm}$. (b): Conductance for a single disorder configuration of a zig-zag (solid blue line) and an armchair (dashed red line) GNR with width $\sim 20 \mathrm{~nm}$ and for $W=0.5$. (c): Configuration averaged (over $\sim 400$ samples) normalized conductance as a function of GNR length for both zig-zag and armchair GNRs. The solid blue (dashed red) arrow shows the energy at which the calculations for the zGNR (aGNR) have been performed. (d) and (e): Same informations as for (b) and (c) but for a larger disorder strength $(W=2)$.

of ideal GNRs with width below $100 \mathrm{~nm}$ and well defined edge symmetries (zig-zag or armchair types) are dominated by confinement effects and Van Hove singularities [10], similarly to carbon nanotubes [11]. Zig-zag type GNRs show very peculiar electronic properties with wavefunctions sharply localized along the GNRs edges at low energies, which will significantly influence their transport properties. In contrast, armchair-type GNRs share more similarities with metallic nanotubes. Some prior studies have already addressed the impact of homogeneous disorder on quantum transport in GNRs [18].

By using a Landauer approach [15, 19], $\ell_{e}(E)$ and $\xi(E)$ can be estimated from the scaling analysis of the average conductance of the GNRs [19]. In particular, $\xi(E)$ can be accurately 
extracted from the exponential decay of the conductance (averaged over $\sim 400$ configurations) versus length.

In Fig. 4, the conductance for both types of symmetries are shown in the ideal case and for a single disorder configuration for weak $(W=0.5)$ as well as strong disorder $(W=2)$. The length scaling behaviors of the conductance and the localization lengths (for both types of GNRs edge symmetries) are respectively reported in Fig. 4k and 4e and in Fig. 3, For disorder as large as $W=2$, the localization lengths are similar for both types of ribbons, showing that edge symmetry does not play any role. In contrast, $\xi$ is up to two orders of magnitude larger in zig-zag than in armchair GNRs in the low disorder limit $(W=$ 0.5), for all values of energy in the plateau around CNP (some illustrative $\xi$ values at chosen energies are shown in Fig. 31). This can be simply understood as the consequence of the lower dimensionality of transport in the case of zig-zag symmetry, driven by more confined wavefunctions [10]. However, $\xi$ remains always several orders of magnitude smaller in GNRs than in 2D graphene, whatever the disorder strength. Similar results are obtained for GNRs with larger width of $\simeq 80 \mathrm{~nm}$ (not shown). This points out the predominant role of dimensionality and edge effects on quantum transport especially in the low disorder limit.

In conclusion, by studying the transport properties in both disordered 2D graphene and GNRs (for short range scattering potential), the impact of edge symmetries and transport dimensionality on the transport length scales was outlined. Despite the simplicity of the Anderson model, some of the reported transport features may be generic to other types of disorder such as chemical doping, surface functionalization, or topological defects. However, the contribution of long range potentials (e.g. due to ionized impurities trapped in an oxide) deserves further consideration.

BB acknowledges the Institute CARNOT for financial support. We thank the CEA/CCRT supercomputing facilities for providing computational resources.

[1] K.S. Novoselov et al., Science 306, 666 (2004). K.S. Novoselov et al., Nature 438, 197 (2005). A.K. Geim and K.S. Novoselov, Nature Materials 6, 183 (2007).

[2] C. Berger et al., J. Phys. Chem. B 108, 19912-19916 (2004). C. Berger et al., Science 312, 1191 (2006). 
[3] M.I. Katsnelson, K.S. Novoselov, A.K. Geim, Nat. Phys. 2, 620-625 (2006). K.S. Novoselov et al., Nat. Phys. 2, 177 (2006). K.S. Novoselov et al., Science 315, 1379 (2007). Y. Zhang, Y.-W. Tan, H. L. Stormer, and Ph. Kim, Nature 438, 201-204 (2005).

[4] Y.W. Tan et al., arXiv:070\%.1807v1.

[5] N. H. Shon and T. Ando, J. Phys. Soc. Jpn. 67, 2421 (1998). Y. Zheng and T. Ando, Phys. Rev. B 65, 245420 (2002). T. Ando, Int. J. Mod. Phys. B 21, 1113 (2007).

[6] E. McCann et al., Phys. Rev. Lett. 97, 146805 (2006). 69 (2002). P. M. Ostrovsky, I. V. Gornyi, and A. D. Mirlin, Phys. Rev. B 74, 235443 (2006). A. F. Morpurgo and F. Guinea, Phys. Rev. Lett. 97, 196804 (2006). D. V. Khveshchenko, Phys. Rev. B 75, 241406 (2007). K. Wakabayashi, Y. Takane, M. Sigrist, Phys. Rev. Lett. 99, 036601 (2007).

[7] H. Suzuura and T. Ando, J. Phys. Soc. Jpn. 72, 69 (2003). A. Altland, Phys. Rev. Lett. 97, 236802 (2006). I. L. Aleiner and K. B. Efetov, Phys. Rev. Lett. 97, 236801 (2006).

[8] S. V. Morozov et al., Phys. Rev. Lett. 97, 016801 (2006). X. Wu et al., Phys. Rev. Lett. 98, $136801(2007)$

[9] M. Y. Han et al., Phys. Rev. Lett. 98, 206805 (2007). M.C. Lemme et al., IEEE Electron Device Letters 28 (4), 1 (2007). Z. Chen et al., Physica E (2007), doi:10.1016/j.physe.2007.06.020

[10] K. Nakada et al., Phys. Rev. B 54, 17954 (1996). K. Wakabayashi et al., Phys. Rev. B 59, 8271 (1999). N. Peres, A. H. Castro Neto, F. Guinea, Phys. Rev. B 73, 195411 (2006). F. Muñoz-Rojas et al., Phys. Rev. B 74, 195417 (2006).

[11] J.C. Charlier, X. Blase, S. Roche, Rev. Mod. Phys. 79, 677 (2007).

[12] K. Ziegler, Phys. Rev. Lett. 97, 266802 (2006); ibidem Phys. Rev. B 75, 233407 (2007). K. Nomura and A. H. MacDonald, Phys. Rev. Lett. 98, 076602 (2007). E. H. Hwang, S. Adam, S. Das Sarma, Phys. Rev. Lett. 98, 186806 (2007). J. Cserti, Phys. Rev. B 75, 033405 (2007).

[13] P.W. Anderson, Phys. Rev. 124, 41 (1961). E. Abraham et al., Phys. Rev. Lett. 42, 136801 (1979). F. Evers and A.D. Mirlin, Rev. Mod. Phys., in press.

[14] P. A. Lee and D. S. Fisher, Phys. Rev. Lett. 47, 882 (1981). P. A. Lee and T. V. Ramakrishnan, Rev. Mod. Phys. 57, 287 (1985). A. MacKinnon and B. Kramer, Phys. Rev. Lett. 47, 1546 (1981).

[15] S. Datta, in Electronic Transport in Mesoscopic Systems, Cambridge University Press (1995).

[16] S. Roche and R Saito, Phys. Rev. Lett. 87, 246803 (2001). F. Triozon et al., Phys. Rev. B 69, $121410(2004)$. 
[17] Periodic boundary conditions are applied. The convergence is achieved for typical supercell sizes $L_{x}=320 \mathrm{~nm}$ and $L_{y}=185 \mathrm{~nm}$, which enclose more than 2 millions carbon atoms.

[18] D.A. Areshkin, D. Gunlycke, C.T. White, Nano Lett. 7, 204 (2007). D. Gunlycke, D. A. Areshkin, C.T. White, Appl. Phys. Lett. 90, 142104 (2007). D. Gunlycke, H. M. Lawler, C. T. White, Phys. Rev. B 75, 085418 (2007).

[19] B. Biel et al., Phys. Rev. Lett. 95, 266801 (2005). R. Avriller et al., Phys. Rev. B 74, 121406 (2006). 